\title{
Brain Tumour Segmentation in FLAIR MRI Using Sliding Window Texture Feature Extraction Followed by Fuzzy C-Means Clustering
}

\author{
Sanjay Saxena, IIIT Bhubaneshwar, India \\ (iD) https://orcid.org/0000-0002-8288-1010 \\ Nitu Kumari, IIIT Bhubaneswar, India \\ Swati Pattnaik, IIIT Bhubaneswar, India
}

\begin{abstract}
In this paper, a hybrid approach using sliding window mechanism followed by fuzzy c means clustering is proposed for the automated brain tumour extraction. The proposed method consists three phases. The first phase is used for detecting the tumorous brain MR scans by implementing preprocessing techniques followed by texture features extraction and classification. Further, this phase also compares the performance of different classifiers. The second phase consists of the localization of the tumorous region using sliding window mechanism, in which a sized window sweeps through the whole tumorous MR scan and the window is classified as tumorous or non-tumorous. The third phase consists of fuzzy c means clustering to get the exact location of the tumour by removing the misclassified windows obtained from Phase 2. 2D single-spectral anatomical FLAIR MRI scans are considered for experiment. Outcomes demonstrate significant results in terms of sensitivity, specificity, accuracy, dice similarity coefficient in comparison with the other existing methods.
\end{abstract}

\section{KEYWORDS}

Brain Tumor Segmentation, Magnetic Resonance Imaging (MRI), Medical Image Analysis

\section{INTRODUCTION}

In recent years, current statistics show a growing number of people suffering from a brain tumor at an alarming pace. As per a 2018 estimate, about 700,000 people in the United States (US) alone are living with a prime brain tumor while over 79,000 more people have been diagnosed with such related conditions ("Quick Brain Tumor Facts",2018). A brain tumor comprises a group of the anomalous cells in the brain with the capability of eliminating brain cells, increasing inflammation in the brain. Owing to the varying shape, size, texture, and position of the tumor, the automated extraction of

This article, published as an Open Access article on December 11, 2020 in the gold Open Access journal, International Journal of Healthcare Information Systems and Informatics (IJHISI) (converted to gold Open Access January 1, 2021), is distributed under the terms of the Creative Commons Attribution License (http://creativecommons.org/licenses/by/4.0/) which permits unrestricted use, distribution, and production in any medium, provided the author of the original work and original publication source are properly credited. 
such brain tumor(s) from the brain Magnetic Resonance Imaging (MRI) sequences is a non-trivial, but pertinent exercise.

Over the last three decades, despite the accumulation of a large number of brain tumor cases, and their devastating prognosis, only four FDA-approved medicine and one equipment for the treatment of patients who have brain tumors have been advocated (Angulakshmi \& Lakshmi Priya, 2017). In fact, there are over 140 varieties of brain tumors; among them, two main categories are: (a) Malignant; v. (b) Benign tumors. For malignant tumors, two sub-categories exist: (i) a primary tumor starting inside the brain; or (ii) a secondary tumor or baptized brain metastasis tumor, which is dispersed from other body parts. Benign tumors are largely a mass of tissues with deficient capability to spread.

Today, a novel automatic method for 2D single-spectral anatomical Fluid Attenuated Inversion Recovery (FLAIR) MR Scans for tumor detection is being proposed. Here, the detection of tumorous MR scans, the process of brain tumor segmentation, and the evaluation of its efficacy have been piloted. For both the detection and localization of tumorous brain MR scans, different texture features, including first-order statistical features (Nabizadeh \& Kubat, 2015), Gray Level Co-occurrence Matrix (GLCM), Gray Level Run Length Matrix (GLRLM), Histogram of Gradient (HOG), Local Binary Pattern (LBP), Cross Diagonal Texture Matrix (CDTM), and Simplified Texture Spectrum Features (STSF) have been deployed. For detecting tumorous brain MR scans, various classifiers have also been compared. For example, a sliding window of specific (45 X 45) size sweeping the entire scanned "tumor" image is considered for brain tumor segmentation via MR scans. Nabizadeh \& Kubat (2015) have experimented with three different-sized windows and found a $45 \times 45$ window to produce the best segmentation results. Moreover, this window or slice may be further classified as tumorous $\mathrm{v}$. non-tumorous using SVM (Support Vector Machine) via linear and radial basis function (RBF) kernel.

The rest of this paper is organized as follows. Section 2 presents the background of the brain MR scans and recent contributions whereas Section 3 summarizes the different types of features considered for feature extraction. Following this, Section 4 presents the fuzzy c-means clustering methodology while Section 5 defines the implementation of the proposed novel technique for brain tumor segmentation. Section 6 deliberates about datasets, results obtained and more elaborated discussions of the findings and associated implications. Finally, Section 7 offers concluding remarks, including chief contributions of this work, its limitations and potential future work.

\section{BACKGROUND}

Medical image processing, via MRI and other means (e.g., CT Scan), plays a key role in the analysis of brain tumors; in turn, this process supports how the tumor and its treatment plan may best be handled. Primarily used in the diagnosis of brain tumor and for treatment planning, the MRI is a powerful non-invasive medical imaging modality for brain scans ("MRI Basics", 2018). It offers a variety of valuable features, including multiplanar capabilities as well as prospective of tissue characterization with no teeth and bone artefacts. Various MR sequences are generated by changing the excitation times during the acquisition of an image (e.g., Clark, Hall \& Goldgof, 1998; Işın, Direkoğlu \& Şah, 2016). These various MRI sequences produce diverse kinds of tissue contrast images, creating very significant basic information to allow the proficient extraction of tumors alongside their sub-regions (e.g., El-Dahshan, Mohsen, Revett \& Salem, 2014; Işın et al., 2016; Saxena, Garg \& Pattnaik, 2019).

Figure 1 portrays the three different MRI sequencing standards for brain tumor diagnosis known respectively as the T1, T2 and FLAIR MRI Sequences (Ahmed, Iftekharuddin \&Vossough, 2011).

Additionally, Table 1 displays the assessment of T1 v. T2 v. FLAIR MR Sequences. Typically, T1 and T2 MR sequences may be easily differentiated by CSF (Cerebro-Spinal Fluid). For example, T1-weighted CSF often produces darker images whereas T2-weighted CSF images are bright. Saxena, Mohapatra \& Pattnaik (2019) note that FLAIR MR sequencing is similar to T2 except that Echo Time (TE) and Repetition Time (TR) times are lengthier ("MRI Basic", 2018). 


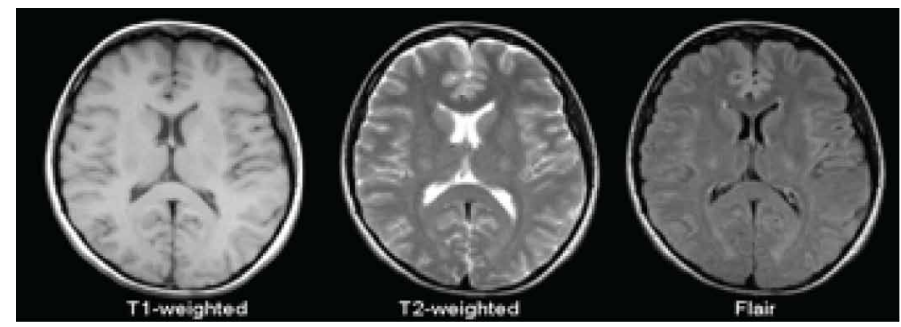

Table 1. The Analysis of Basic MRI Sequences (Brain)

\begin{tabular}{|l|l|l|l|}
\hline \multicolumn{1}{|c|}{ Tissue } & \multicolumn{1}{c|}{ T1-Weighted } & \multicolumn{1}{c|}{ T2-Weighted } & \multicolumn{1}{c|}{ FLAIR } \\
\hline White Matter & Light & Dark Gray & Dark Gray \\
\hline CSF & Dark & Bright & Dark \\
\hline Cortex & Gray & Light Gray & Light Gray \\
\hline Fat (Within bone marrow) & Bright & Light & Light \\
\hline Inflammation & Dark & Bright & Bright \\
\hline
\end{tabular}

In MR Scans, sometimes it is difficult to distinguish among precise tissues and cells from the respite of the image (Nabizadeh \& Kubat, 2015); hence, image segmentation is implemented either manually or automatically. Essentially, image segmentation divides an image into a group of the corresponding homogeneous area with related properties. Manual image segmentation is time intense and complex for a large volume of MR scans (Aslam, Khan \& Beg, 2015). Hence, an automatic means of segmenting brain MR scans is essential for the efficient localization of a brain tumor within a reasonable time in order to achieve a superior treatment plan. Nonetheless, several techniques have emerged for automated brain tumor segmentation, for example, thresholding, edge-based method, morphological image processing, watershed segmentation, atlas-based method, deformation model, graph cut methods, among others (Angulakshmi \& Lakshmi Priya, 2017). Even so, accurate segmentation remains challenging for many researchers. As noted, the published literature for brain tumor segmentation have pervaded in recent years.

\subsection{Recent Contributions}

Incorporating the review of Kumari \& Saxena (2018), this section overviews recent works with advantages and limitations on different techniques of brain tumor segmentation and classification.

Sudharani, Sarma \& Prasad (2016) have proposed a method to extract the tumorous region in low-intensity MR scans. Their approach is to apply a series of steps to segment tumorous regions such as image enhancement, colour plane extraction, re-sampling of the image, among others. Image morphology is primarily implemented as the filter to exterminate pixels of low-frequency and the picture elements present in the border region. Diverse parameters of the tumor such as length and area are efficiently demarcated for diagnosis and treatment. The core downside of this method is that it uses several monotonous steps for the segmentation. In (Pingale \& Todmal, 2018), the authors have modelled Fuzzy C-Means (FCM) and k-means on the T1 contrast axial plane MRI scans for the extraction of the tumorous region through the histogram steered initialization of the cluster. K-means algorithm is more proficient than FCM although FCM recognizes scarcely classes of three tissues. Notwithstanding, K-means recognizes every six classes with the major shortcoming being that few white matter (WM) may be categorized as edema and vice versa. 
Al-Shaikhli, Yang \& Rosenhahn (2014) have implemented the topological graph prior information of atlas in a bespoke multilevel set invention for multiregional localization of brain tumorous MRI scans. The drawback here is that accuracy has to be a priori contingent on the accurateness of the topological graph. As well, Pereira, Pinto, Alves \& Silva (2016) have suggested Convolutional Neural Network (CNN) based classifier with the normalization of intensity and patch, a novel deep learning method used for brain tumor extraction and classification. Here, data expansion is used to accomplish the capriciousness of structural configuration and spatial localization of the brain tumor. In (Aslam, Khan \& Beg, 2015), the authors promote a method based on closed contour algorithm, namely, the Sobel edge detection and thresholding techniques. Here, the segmented images of brain tumors are well equated to normal Sobel edge detection in terms of dice similarity coefficients. Additionally, there is the scope of improvement for the closed contour method to upsurge the province while reducing the thickness of the borderlines of the different regions.

Havaei, Dutil \& Pal (2016) have used CNN techniques to achieve high performance via a novel two-way architecture by positioning two CNNs instead of relying on local label dependencies. In (Priya \& Shobarani, 2016), contextual clustering technique, which delivers improved segmentation correctness by plummeting false segmentation, have been used. A future possibility is to amend the value of variables used in the proposed method for further enhancement in the segmentation of brain tumor. Subbanna, Precup \& Arbel (2014) have developed an iterative Marcov Random Field (MRF) framework to comprise voxel-based MRF, adopted MRF, and regional MRF. The implemented context has also been used to classify all the subclasses of the brain MR sequences. The chief constraint of MRF is its computational complexity and effective selection of parameters even though it is regularly used to model belongings of texture and intensity inhomogeneity. In (Menze et al., 2015), generative-discriminative model is implemented to convey the set-up and results of the multimodal Brain Tumor Segmentation (BraTS) challenge held in grouping with the MICCAI 2012 and 2013 conferences. Numerous tumor segmentation algorithms have been applied to a set of 65 multi-contrast MR scans of Low-Grade Glioma (LGG) v. High-Grade Glioma (HGG) patients via hand-annotated and to 65 similar scans produced via tumor image simulation software. Finally, Madhukumar \& Santhiyakumari (2015) have implemented contrast limited adaptive histogram equalization, K-means and FCM, demonstrating that k-means distinguish CSF fluid, grey matter and white matter regions reasonably better than FCM.

Other notable related works include that of Işın et al. (2016), who have developed a Deep CNN model for tumor segmentation. Key features here include the efficient handling of an enormous volume of MR slices. Dahab, Ghoniemy,Gamal \& Selim (2012) employ customized probabilistic neural network (PNN) with linear vector quantization (LVQ) modelling procedure for the classification of meticulous region-of-interest (ROI). The extraction of the feature's sets is done from every ROI to estimate brain tumor, assigning a mass. These allocated weights are cast-off for modelling network based on LVQ. With Nabizadeh \& Kubat (2015), segmentation, tumor detection and assessment of Statistical and Gabor feature consuming numerous classifiers (K-NN, SVM, K-Means and many more) are evaluated, showing the effectiveness of statistical features in terms of higher accuracy with smaller dimensionality and no need for the initial assumption. In Jaffar, Naveed \& Ahmed (2009), anisotropic diffusion filtering, FCM methods are implemented for detecting and extracting the average DSC (Dice Similarity Coefficients) obtained being 0.729, which stretches the efficient segmentation and detection of a brain tumor in MR sequences. In future, it is noted that circularity criteria may be used with other feature extraction technique to enhance its efficiency. Sonavane \& Sonar (2016) also implement Ada Boost classifier (Islam, Reza \& Iftekharuddin, 2013), Edge Detection, Anisotropic Diffusion Filtering, Stationary Wavelet Transform, with implementation yielding a perfect score $(100 \%)$ correctness on 155 MR sequences. It is noted that their implementation actually delivers an automatic system with improved correctness in minimum time to detect a brain tumor.

Last, but not the least, SVM ensemble base classifier techniques are implemented by Ain, Jaffar \& Choi (2014) for handling texture features. Here, tremendous precision of the classification 
(greater than 99\%) has been accomplished with the possibility of measuring the depth and area of the tumor extracted region in future. As well, Abdel-Maksoud, Elmogy \& Al-Awadi (2015) report using K-means clustering, mean shift, expectation-maximization and FCM. The benefit of their approach is the expansion of a novel method that syndicate the k means with the FCM for detecting brain tumor with advanced accuracy within least timeframe. In (Soltaninejad, Yang \& Lambrou, 2016), aside from Super-pixel, the extremely randomized trees (ERT) classifier, SVM and Gabor Texton feature techniques are used for brain tumor segmentation. The advantage of this integrative approach is indicative of an entirely automatic technique to perceive and extract the brain tumor from FLAIR MR sequences by calculating the Gabor text function, curvature, fractal analysis and statistical intensity characteristics from super-pixels. According to the authors, Super-pixel grounded enormously randomized trees in FLAIR can also slog for T1 and T2 type MR sequences. In contrast, Telrandhe, Pimpalkar \& Kendhe (2016) employ K-means clustering algorithm, object labelling algorithm, with SVM classification methods for brain tumor segmentation. The major benefit of their approach is that it delivers adaptive brain tumor recognition. Intended for making the segmentation process adaptive, this proposed system deploys SVM in an unsupervised style, thereby stretching better result relatively to other prevailing methods. In future, the approach may be extended for handling 3-dimensional image data.

More recently, Lahmiri (2017) reports on different classification techniques such as particle swarm optimization, and SVM. Experimental consequence displays that feature abstraction from Fractional Order Darwinian PSO (FODPSO) segmented images provides advanced performance rather than the classical DPSO and PSO. Sachdeva, Kumar, Gupta, Khandelwal \& Ahuja (2016) have proposed content-based active contour to segment brain tumors with extracted features being highdimensional. To diminish those extracted features, Genetic Algorithm (GA) is implemented. Here, GA with ANN and GA with SVM are implemented for brain tumor segmentation and classification with results compared. GA with SVM yields superior processing speed, whereas GA with ANN produces better accuracy. Again, the issue here is that computational complexity increases due to hybridization. Hence, it appears that despite the multiple emerging and promising techniques and methods developed for brain tumor detection, research for developing an automated system to achieve accurate tumor segmentation in a reasonable time is still seriously lacking.

\section{METHODS FOR TEXTURE FEATURES EXTRACTION}

Several texture features extraction techniques such as first-order statistical features, GLRLM, GLCM, HOG, LBP features are summarized herein. Further, Cross Diagonal Texture Features (CDTM) and Simplified Texture Spectrum (STS) are also implemented to achieve even higher accuracies.

\subsection{First Order Statistical Features}

Certain useful features of first-order statistical features include mean, energy, entropy, average contrast, skewness and kurtosis.

Mean is the average value of the intensity of the image. Variance specifies the variations of intensities about the mean value. Skewness enumerates the unevenness of the histogram around the mean value. Kurtosis denotes the flatness of the histogram. Entropy reveals the haphazardness of different intensity values. Formulas are listed below for these features as given in Table 2 (Nabizadeh \& Kubat, 2015).

\subsection{Grey Level Co-Occurrence Matrix (GLCM) Features}

Basically, GLCM describes a statistical method of analysing texture information that deliberates the spatial relationship of the pixels. It evaluates the properties of the image that belong to $2^{\text {nd }}$-order statistics, representing the association of pair of pixels. 
Table 2. First Order Statistical Features

\begin{tabular}{|c|c|}
\hline Mean: & $\mu=\sum_{i=0}^{g-1} i p r(i)$ \\
\hline Average contrast & $\sigma^{2}=\sum_{i=0}^{g-1}(i-\mu)^{2} \operatorname{pr}(i)$ \\
\hline Skewness & $\mu_{3=\sigma^{-3}} \sum_{i=0}^{g-1}(i-\mu)^{3} \operatorname{pr}(i)$ \\
\hline Kurtosis & $\mu_{4=\sigma^{-4}} \sum_{i=0}^{g-1}(i-\mu)^{4} \operatorname{pr}(i)-3$ \\
\hline Energy & $E=\sum_{i=0}^{g-1}[p r(i)]^{2}$ \\
\hline Entropy & $H=-\sum_{i=0}^{g-1} p r(i) \log _{2}[p r(i)]$ \\
\hline \multicolumn{2}{|c|}{$\begin{array}{l}\text { where } \mathrm{g} \text { is representing the image maximum gray level and } \operatorname{pr} \text { (i) is the probability density of the intensity levels } \\
\text { which is calculated by: } \\
\operatorname{pr}(\mathrm{i})= \\
\text { Where } \mathrm{hl}(\mathrm{i}) \text { is the whole numeral of pixels of intensity level } \mathrm{i} \text { and } \mathrm{T} \text { is the whole numeral of pixels present in the image. }\end{array}$} \\
\hline
\end{tabular}

Generally, $\mathrm{d}=1,2$ and $\theta 0^{\circ}, 45^{\circ}, 90^{\circ}, 135^{\circ}$ are used for calculation (Joshi, Rana \& Misra, 2010; Nabizadeh \& Kubat, 2015). The following are the eight different texture features that are represented by the co-occurrences matrix as given in Table 3 .

\subsection{Gray Level Run Length Method (GLRLM) Features}

GLRLM is also a $2^{\text {nd }}$-order statistical method that gives a significant parameter to a spatial field grey level value. $R(\theta)$ is calculated for $\theta 0^{\circ}, 45^{\circ}, 90^{\circ}, 135^{\circ}$. The following five features, as shown in Table 4, are computed for GLRM features (Ahmed, Iftekharuddin \&Vossough, 2011).

\subsection{Histogram of Oriented Gradient Features (HOG)}

HOG descriptors are used to detect objects in computer vision and image processing. The technique is to count the gradient orientation in confined parts of an image or area of concentration. For HOG, first divide an image into small cells; then, individually cell histogram of gradient positioning is calculated for every pixel within the cells. Combining these histograms yields a descriptor ("Histogram of Oriented Gradients", 2018). 
Table 3. GLCM Features

\begin{tabular}{|c|c|}
\hline Entropy & $-\sum_{i=0}^{G-1} \sum_{j=0}^{G-1} C_{i j} \log _{2} C_{i j}$ \\
\hline Correlation & $\sum_{i=0}^{G-1} \sum_{j=0}^{G-1} \frac{i j C_{i J}-\mu_{x} \mu_{y}}{\sigma_{x} \sigma_{y}}$ \\
\hline Homogeneity & $\sum_{i=0}^{G-1} \sum_{j=0}^{G-1} \frac{C_{i J}}{1+|i-j|}$ \\
\hline Absolute value & $\sum_{i=0}^{G-1} \sum_{j=0}^{G-1} C_{i J}|i-j|$ \\
\hline Inertia (contrast) & $\sum_{i=0}^{G-1} \sum_{j=0}^{G-1}(i-j)^{2} C_{i J}$ \\
\hline Inverse difference & $\sum_{i=0}^{G-1} \sum_{j=0}^{G-1} \frac{C_{i J}}{1+(i-j)^{2}}$ \\
\hline Maximum probability & ${ }_{i j}^{\max } C_{i j}$ \\
\hline Angular second moment (energy) & $\sum_{i=0}^{G-1} \sum_{j=0}^{G-1}\left(C_{i j}\right)^{2}$ \\
\hline
\end{tabular}

\subsection{Local Binary Pattern (LBP) Features}

The LBP operator moves a window across the image and provides tags to the central pixels of the window by thresholding its adjacent, describing the binary number for its neighbours with central value as shown in Table 5.

Here, the notation $(\mathrm{P}, \mathrm{R})$ is used for adjacent pixels where $\mathrm{P}$ is a sampling point on the circle of $\mathrm{R}$ radius. Table 5 shows the LBP code of a pixel $\left(\mathrm{x}_{\mathrm{c}}\right.$, yc) (Nabizadeh \& Kubat, 2015).

\subsection{CDTM Features}

For CDTM, the eight (8) elements present in the unit of texture attained from a neighbourhood of 3 $X 3$ pixels are alienated into two clusters, each with four (4) elements.

The elements present at diagonal are organized in a single group, while the further group comprises the other elements present in the unit of texture. Each element in the two different clusters has one of the three probable values with the pattern of 0,1 , and 2 . A " 0 " value is assigned if the value of the intensity of that element is fewer than the central pixel's value, " 1 " if they are identical, and " 2 " if the intensity value is larger than the central pixel's value.The properties of the merger of all four input elements in every cluster produces $81\left(3^{4}\right)$ texture units in overall (Al-Janobi, 2001). These twofold new texture units are called cross-texture unit (CTU) and diagonal-texture unit (DTU), respectively. 
Table 4. GLRLM Features

\begin{tabular}{|c|c|}
\hline Short Run Emphasis (SRE) & $R F_{1}(R(\theta))=\frac{1}{T_{P}} \sum_{i=0}^{G-1} \sum_{l=1}^{N_{R}} \frac{r^{\prime}(i, l / \theta)}{l^{2}}$ \\
\hline Long Run Emphasis (LRE) & $R F_{2}(R(\theta))=\frac{1}{T_{P}} \sum_{i=0}^{G-1} \sum_{l=1}^{N_{R}} j^{2} r^{\prime}(i, l / \theta)$ \\
\hline Gray Level Distribution (GLD) & $R F_{3}(R(\theta))=\frac{1}{T_{P}} \sum_{i=0}^{G-1}\left[\sum_{l=1}^{N_{R}} r^{\prime}(i, l / \theta)\right]^{2}$ \\
\hline Run length Distribution (RLD) & $R F_{34}(R(\theta))=\frac{1}{T_{P}} \sum_{i=0}^{N_{R}}\left[\sum_{l=1}^{G-1} r^{\prime}(i, l / \theta)\right]^{2}$ \\
\hline Run Percentage (RP) & $R F_{5}(R(\theta))=\frac{1}{T_{P}} \sum_{i=0}^{G-1} \sum_{l=1}^{N_{R}} r^{\prime}(i, l / \theta)$ \\
\hline \multicolumn{2}{|c|}{$\begin{array}{l}\text { in which } \mathrm{G} \text { is the number of gray levels, } \mathrm{N}_{\mathrm{R}} \text { is the numeral of run lengths present in the matrix, and } \mathrm{T}_{\mathrm{P}} \text { is } \\
T_{P}=\sum_{i=0}^{G-1} \sum_{l=1}^{N_{R}} r^{\prime}(i, l / \theta), r^{\prime}(i, l / \theta) \text { provides no of epochs an image comprises a run with the length } 1 .\end{array}$} \\
\hline
\end{tabular}

$$
\begin{aligned}
& L B P_{P, R}=\sum_{p=0}^{P-1} S\left(g_{p}-g_{c}\right) 2^{p} \\
& S(x)=\left\{\begin{array}{cc}
1 & \text { if } x \geq 0 \\
0 & \text { Otherwise }
\end{array}\right\}
\end{aligned}
$$

Where $g$ represents the value of pixel intensity and here $\mathrm{P}=8$ and $\mathrm{R}=1$.

\subsection{Simplified Texture Spectrum (STS)}

Benefit of the texture spectrum method is that the texture characteristics of an image are considered by the equivalent texture spectrum rather than texture features because texture spectrum can be reliably used for image analysis and image classification. STS characterizes local texture information in four (4) directions instead of all eight (8) directions as in texture spectrum features. With the STS, the differences of grey-level among the central pixel and its corresponding four (4) neighbours are shortened to three $(3)$ different situations $(=,<$, and $>)$. All the probable texture units are wellorganized in a system of 3 -tuple i.e. $3^{4}=81$ different texture unit such that comparable textural states could have outcome in a very dissimilar unit of texture numbers, which equals: 
with $\mathrm{i}=1,2,3$, and 4, individually, for the four directions and with $\mathrm{Ei}=0,1$, or 2, that resembles individually, the condition of $<,=$ and $>$ among the central pixel and its neighbouring picture element.

In this approach, we do not discriminate the order of the four (4) different neighbourhood pixels in cataloguing the texture unit numeral. This will cluster all the different 81 texture units into only 15 probable states.

\subsection{Feature Aggregation}

First-order statistical features emphasize a total of six (6) features, namely, mean, average contrast, entropy, energy, skewness and kurtosis.

In our experiment, GLCM features are extracted by smearing the angle $0,45,90 \& 135$ and for distance $1 \& 2$. In respective orientation, GLCM matrix and eight (8) derived features (entropy, correlation, homogeneity, absolute value, inertia, inverse difference, maximum probability and energy) are computed, resulting in sixty-four (64) features. GLRLM features are also computed for different angles such as 0, 45, 90 \& 135. Extracted features include Short Run Emphasis (SRE), Long Run Emphasis (LRE), Gray Level Distribution (GLD), Run length Distribution (RLD), Run Percentage (RP) in four (4) directions, yielding a total of 20 features.

HOG features measure the incidences of gradient orientations in the provincial zones of the image. By using two (2) scales and eight (8) different orientations, eighty HOG feature values are computed. The dimension of LBP features is 256. The six (6) CDTM features to be extracted include Homogeneity, Entropy, Absolute value, Contrast, Energy and Inertia Difference Moment. Finally, fifteen (15) STS features are extracted (e.g., Işın et al., 2016; Havaei et al., 2016; Priya \& Shobarani, 2016).

The total of six (6) first-order statistical features, twenty (20) GLRLM features, sixty-four (64) GLCM features, eighty (80) HOG features, two fifty-six (256) LBP features, six (6) CDTM features and fifteen (15) STS features, makes an accumulation of 447-dimensional statistical feature vector. Main reason of considering of such dimensional vector to improve the accuracy of the obtained results of segmentation and classification.

\section{THE FUZZY C-MEANS (FCM) TECHNIQUE}

An unsupervised clustering method used for feature analysis, clustering, and classifier design in medical imaging, segmentation, and more such as astronomy (Chuang, Tzeng, Chen, Wu \& Chen, 2006), FCM categorizes the images which are arranged into similar element within the feature space and grouped into different clusters. FCM method, in our experiment, is used to eliminate the misclassified window obtained via the sliding window technique during the tumor localization step; importantly, it is the distance of the pixel from the cluster centre in the feature space that will determine how the clustering cost may be diminished (e.g., Elnakib, Gimel'Farb, Suri \& El-Baz, 2011; Liew A-C \& Yan, 2003).

\subsection{Centroid}

Consider point $\mathrm{x}$ having a group of the coefficients to accommodate the degree of existence in the $\mathrm{k}^{\text {th }}$ cluster $\mathrm{w}_{\mathrm{k}}(\mathrm{x})$. By FCM, the centroid cluster represents the mean of all-encompassing data elements wholistically, biased by their degree-of-fitting to the cluster:

$$
C_{K}=\frac{\Sigma_{x} w_{k(x)^{m} x}}{\sum_{x} w_{k(x)^{m}}}
$$


where $m$ is the hyper-parameter that controls how fuzzy the cluster will be, that is, a higher $m$ value essentially infers a fuzzier cluster.

\subsection{Method}

The FCM attempts to divide a finite group of:

$\mathrm{n}$ item $X=\left\{x_{1}, \ldots x_{n}\right\}$

into a group of c fuzzy clusters with esteem to some specified standard.

Given a limited group of data, the algorithm yields a list of \{\displaystyle c \}c cluster centres $C=\left\{c_{1}, \ldots c_{n}\right\}$ and a particular partition matrix:

$W=w_{i, j} \in[0,1], i=1, \ldots, n, j=1, \ldots, c$

where, each element, $w_{i, j}$ shows the degree to which element, $x_{i}$ belongs to cluster $c_{j}$ and $\mathrm{m}$ determines the level of cluster fuzziness.

FCM aims to minimize an objective function:

$\arg _{\mathrm{c}} \min \sum_{i=1}^{n} \sum_{j=1}^{c} w_{i j}^{m} m_{x}-c_{j}^{2}$

where:

$$
w_{i, j}=\frac{1}{\sum_{k=1}^{c}\left(\frac{x_{i}-c_{j}}{x_{i}-c_{k}}\right)^{\frac{2}{m-1}}}
$$

\section{GENERAL STRUCTURE OF THE PROPOSED FRAMEWORK}

As shown in Figure 2, the proposed framework comprises two stages: (a) Tumor slice detection; and (b) Tumor localization.

There are three phases applicable in deploying the proposed framework: Phase 1 is for detecting tumorous brain MR slices while Phase 2 is to segment the tumor from the tumorous brain MR slice, followed by Phase 3. We can say that Phases 2 and 3 are sequential. Supposedly, we already have tumorous slice; then, there is no need to go through Phase 1. Instead, we can start from Phase 2 followed by Phase 3. The description of each block is now given below.

\subsection{Tumor Slice Detection}

In tumor slice detection, a key pre-processing step is to implement a median filter to remove poison noise followed by extracting the aforementioned features. Principal Component Analysis (PCA) for dimensionality reduction and classification via supervised vigorous classification methods may then be applied and results compared. The classification techniques can include SVM, KNN (distance = Euclidean, City-block and Minkowski \& $\mathrm{k}=1,3,5,7$ ), Binary Decision Trees, Random Forest and 




Ensemble methods, for example, Adaboost, Gentleboost, Logitboost, LPboost, Robustboost, Rusboost and Totalboost. Aside from these approaches, deep learning CNN model may also be deployed as in the current case being reported (Saxena, Paul, Garg, Saikia \& Datta, 2020).

As shown in Figure 3, training samples are being randomly selected. Accordingly, the feature set's appropriateness for the segmentation of the tumor should be validated. In our case, a 10-fold cross-validation has been implemented to validate the sturdiness of our developed model while averting having to over-fit the data. Subsequent to training the classifier, the rate of recognition of the classifier on independent data is used as the basis to track performance in brain tumor extraction vis-à-vis our proposed model.

\subsection{Tumor Localization}

After detecting the tumorous slice, the localization of the tumor region via a sliding window is implemented. The window moves around the entire brain slice which consists of brain tumors. There are a series of steps in the tumor localization process.

First, a training data set of $45 \times 45$ window size of 100 images representing both healthy v. nonhealthy brain tissues are generated. Hereto, texture features are applied onto this window and are being classified via SVM with a linear kernel. Second, for every instance of window feature set classified via SVM with the training data set, features are being computed from every occurrence of window (size $45 \times 45$ ) throughout the tumor slice. Third, if the window is categorized as tumor-positive, then the centre pixel of the window will be labelled as tumorous; conversely, if the window is categorized as healthy, then the centre pixel of the window is labelled as healthy.

Figure 4 shows the entire series of steps, where the overlapping window of the dimension $45 \mathrm{X}$ 45 are classified as tumorous v. non-tumorous groupings.

\subsection{Performance Measure}

Performance measures, including sensitivity, specificity, accuracy and diice similarity coefficient, are defined as: 
Figure 3. Different Implemented Classification Techniques

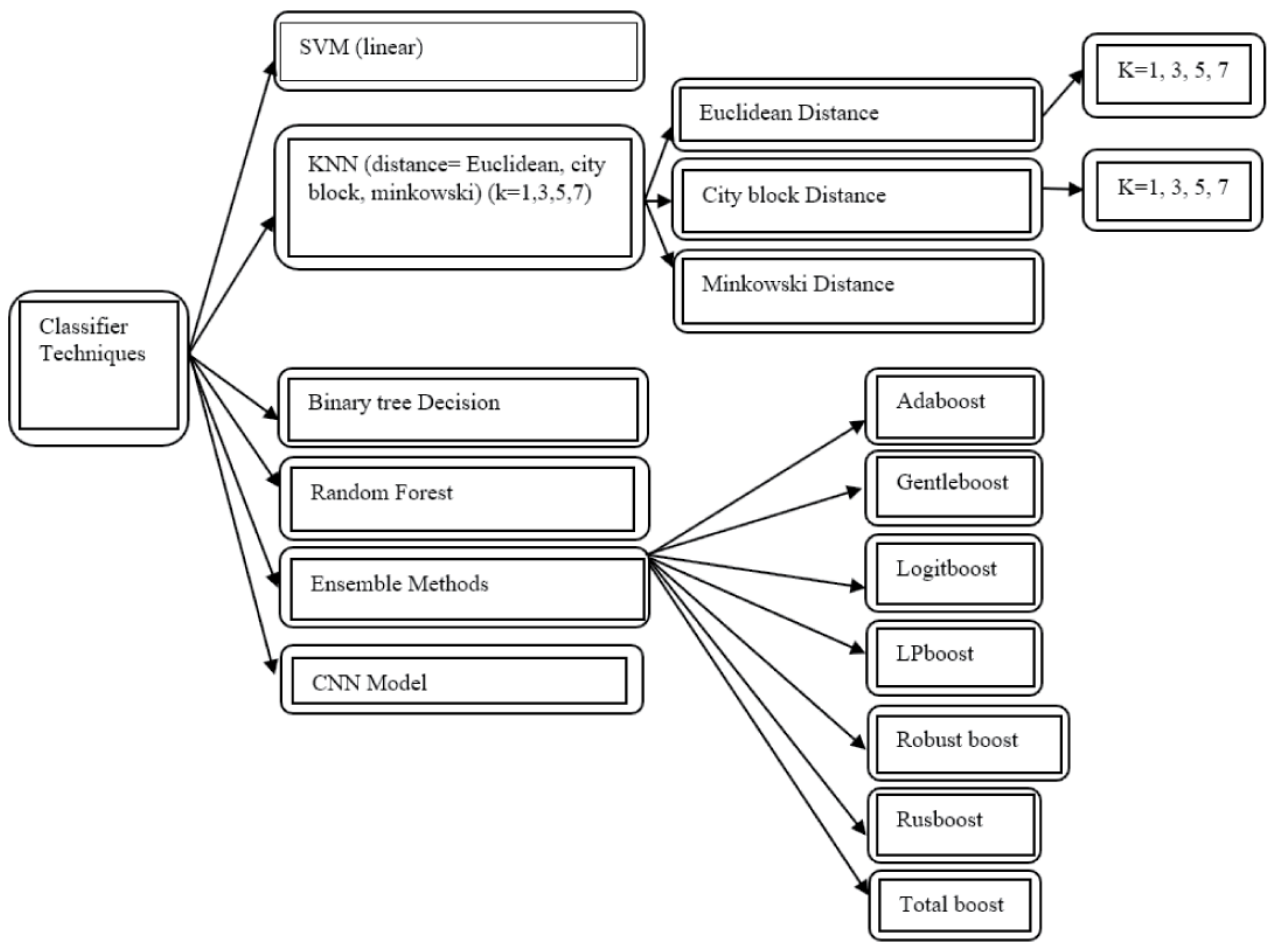

Figure 4. Representation of the implemented system used for localization of brain
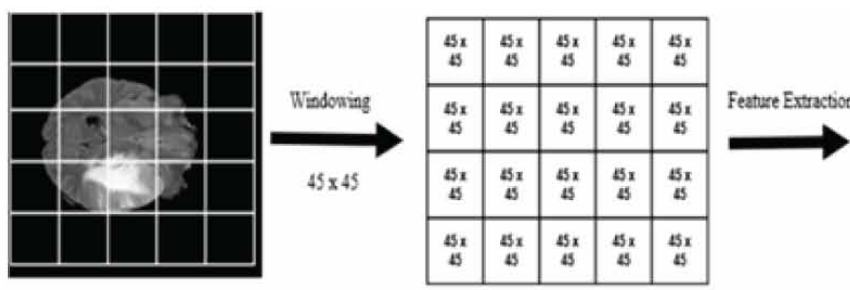

\begin{tabular}{|l|l|l|l|l|}
\hline 447 & 447 & 447 & 447 & 447 \\
\hline 447 & 447 & 447 & 447 & 447 \\
\hline 447 & 447 & 447 & 447 & 447 \\
\hline 447 & 447 & 447 & 447 & 447 \\
\hline
\end{tabular}

Image

Sensitivity $=\frac{t p s}{t p s+f n s} 100 \%$

Specificity $=\frac{t n s}{t n s+f p s} 100 \%$

Accuracy $=\frac{t p s+t n s}{t p s+t n s+f p s+f n s} 100 \%$

where:

tps $=$ Accurately classified positive samples tns $=$ Accurately classified negative samples fps $=$ Inaccurately classified positive samples fns $=$ Inaccurately classified negative samples

Dice similarity coefficient (DSC): The DSC of two sets A and B is expressed as:

$\operatorname{dice}(\mathrm{A}, \mathrm{B})=2 * \mid$ intersection $(\mathrm{A}, \mathrm{B}) \mid /(|\mathrm{A}|+|\mathrm{B}|)$

\section{DATASETS, RESULTS OBTAINED AND DISCUSSION}

\subsection{Data Set}

We have used brain dataset obtained from the NCI-MICCAI 2017 challenge on Multimodal Brain Tumor Segmentation (Menze et al., 2015). NCI-MICCAI 2017 database comprises entirely anonymized images from the institutions: the University of Bern, University of Debrecen, ETH Zurich and University of Utah and also it is publicly accessible images from The Cancer Imaging Archive (TCIA). We have used HGG (High-Grade Glioma) and LGG (Low -Grade Glioma) of 285 subject patients. There were 4 types of brain MR sequences T1, T2, T1ce, FLAIR. We have considered only FLAIR brain MR scans.

\subsection{Brain Tumor Slice Detection}

This process includes three steps depicted in Figure 2. In the first step for pre-processing, we have used a median filter to remove noise from the image. In the second step, above-mentioned features extracted from MR data and applied PCA for feature reduction. Then in third step classification through SVM with linear and RBF kernel is implemented to identify tumorous and healthy MR slices. For the detection, 400 FLAIR tumorous and non-tumorous brain MR scans are considered after implementing 10-fold cross-validation. Further for brain tumor slice detection, a deep model is also implemented for comparing the accuracy of the deep model with the other machine learning technique. Deep learning is a subset of the machine learning field which gained a lot of interest by the researchers in the last several years. 
We have implemented a CNN (Convolutional Neural Network) model that consists convolution layer, ReLU (Rectified Linear Unit) layer, pooling layer (Naceur, Saouli, Akil \& Kachouri, 2018). Firstly, the convolution layer is applied then different sensitivity of the filter is sub-sampled. Further, the transfer of signal from one to another layer is controlled by the activation layer. Then relu layer is used. We have used small kernels for the architecture and got $96.7 \%$ training accuracy. Further, we got high validation accuracy and low validation loss. We can conclude that the CNN model gives the best accuracy in brain tumor slice detection. However, the overall time complexity in the case of CNN model is higher than the other. Figure 5 demonstrates the accuracy obtained from different classifiers.

\subsection{Brain Tumor Localization}

After implementing the above steps as given in section 5.2 following results are obtained. Figure 6 shows the tumor localization of three sample brain slices using statistical features and SVM with a linear kernel. Figure 6(a) shows that some misclassification of the pixels is there which means some non-healthy pixel mistakenly labelled as healthy pixel and some healthy pixel mistakenly labelled as non-healthy pixels. So, some tumor part is labelled as healthy. Further, in the fourth step for removing misclassified pixels fuzzy c- means clustering technique has been implemented as described in section 4. For the better results, we also applied some morphological operations (erosion, dilation) on the segmented MR scans. Figure6(c) shows the segmented tumor after removing the misclassified window. Table 6 shows the result of SVM with a linear kernel for statistical feature extracted from FLAIR images of data set it gives $97.89 \%$ accuracy. To classify each window SVM with a linear

Figure 5. Statistical analysis of the different classifiers used in brain tumorous slice detection, where d denotes the distance

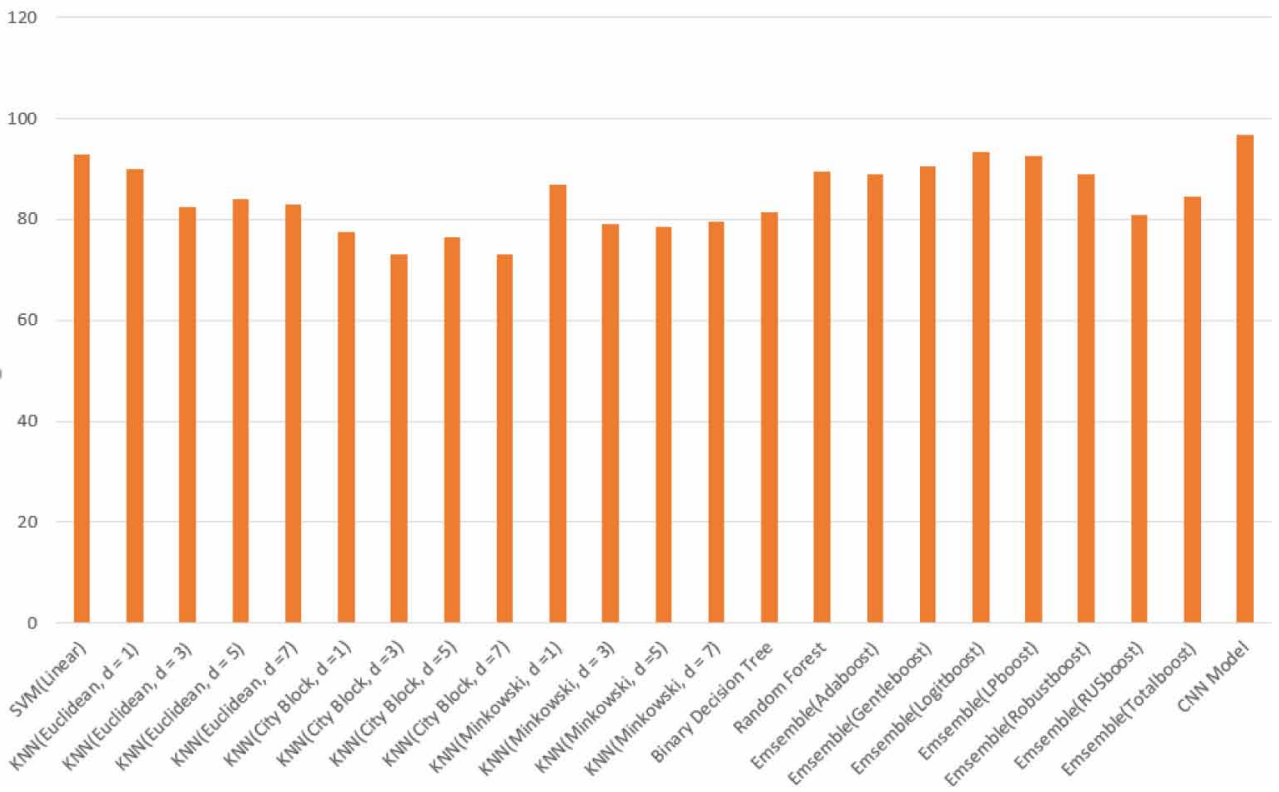

Table 6. SVM classifiers accuracy of FLAIR dataset of tumor localization steps (Average after 10 cross-validations)

\begin{tabular}{|l|l|l|l|}
\hline \multicolumn{1}{|c|}{ SVM } & \multicolumn{1}{|c|}{ Sensitivity } & \multicolumn{1}{c|}{ Specificity } & \multicolumn{1}{c|}{ Accuracy } \\
\hline Linear & 97.87 & 97.91 & 97.89 \\
\hline
\end{tabular}


Figure 6. Examples of tumor segmentation on FLAIR data. (a) brain MR scan (b) segmented tumor using sliding window segmented tumor after fuzzy c-means for misclassification of pixels (c) overlay of our segmented area on the brain image (d) region outline of our segmented region on the brain image (e) Tumor Outlined Results
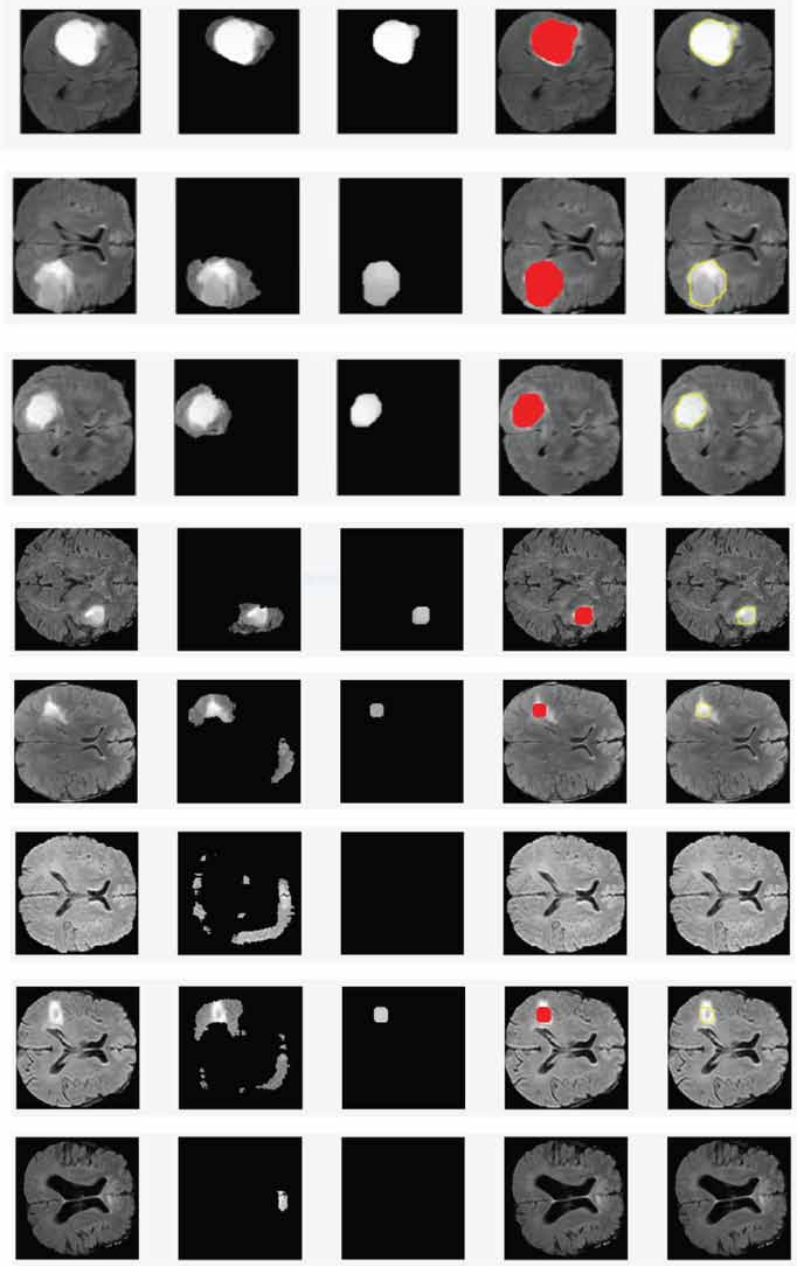

kernel is used. Table 6 depicts the classification accuracy of SVM with the linear kernel which is implemented for classifying window as tumorous and non-tumorous.

\subsection{Dice Similarity Coefficient (DSC)}

It is used for comparing the similarity between two data sample. Here we have compared similarity between ground truth segmented image and automatically segmented image. The following graph as shown in Figure 7 is plotted for 100 tumorous FLAIR brain MR scans of DSC. That is found to be lying between 0.851 to 0.982 . For perfect matching, it should be 1 . DSC lies between 0 to 1 . Zero means no similarity and 1 is for a perfect match.

\subsection{Discussion}

In existing current literatures, many techniques are used for the accurate segmentation of brain tumor such as improved edge detection (Aslam et al., 2015), super-pixel based ERT (Soltaninejad, Yang \& Lambrou, 2016), fuzzy c-means (Kumari \& Saxena, 2018), CNN based segmentation (Pereira, 




Pinto, Alves \& Silva, 2016). Our experimental result is comparable, and, in some cases, it is better than with different proposed method applied by different researchers. In (Soltaninejad et al., 2016) there is an average dice score is 0.88 for BRATS dataset also in (Jaffar et al., 2009) the average dice coefficient is 0.729 and, in our experiment, the average dice coefficient is 0.921 for BRATS FLAIR dataset. The 0.921 dice score shows the close match of ground truth segmented image with our automatic segmented tumor region. The classification accuracy in (Ain et al., 2014) is more than 99\% for segmentation of tumors. The accuracy of our system is in tumor detection step using several classifiers are significant and in tumor localisation step it is lying between 0.851 to 0.98 . For tumor localization in (Nabizadeh \& Kubat, 2015) the SVM with linear kernel classification accuracy is $95.1 \%$ for HGG. In this work, the BRATS data set is used for evaluation and testing which is easily publicly available. FLAIR dataset has been used in our system, it is $97.89 \%$ accurate using statistical feature extraction. Recently, deep learning plays a very vital role in the area of brain tumor segmentation and classification. In (Alqazzaz, Sun, Yang \& Nokes, 2019) authors have used deep learning-based fully convolutional network (SegNet) for a brain tumor on multi-model brain MR images. They have used four MRI modalities such as T1, T2, T1ce and FLAIR. They have also used BRATS 2017 data set for the analysis. They have achieved maximum of 0.85 F-Measure scores for the whole tumor. Though, they have used several multimodalities MR scans. However, on average, our obtained result is quite comparable with their result. In (Havaei et al., 2017), authors have implemented a deep neural network for brain tumor segmentation and tested different architecture. Further, in (Abd-Ellah, Awad, Khalaf \& Hamed, 2018) authors have implemented a two-phase multi-model automatic diagnosis system for brain tumor detection and localization. The authors have achieved dice score 0.87 for brain tumor localization phase while evaluating using 804 3D MRIs from the BRATS 2013 database. This also shows that our proposed method is quite comparable $\&$ better in terms of accuracy and dice value with the other existing methods as well as deep learning-based approaches for brain tumor segmentation. 


\section{CONCLUSION}

Altogether, a fully automated system is developed for diagnosing the brain tumor from brain MR images. This integrated automated framework can detect FLAIR MR images containing tumor and then segmentation of the tumorous region. This system performs the diagnosis in three phases. The first phase is used for detecting the tumorous brain MRI. The second phase consists of the localization of the tumorous area using a sliding window mechanism followed by fuzzy c means. The remarkable accuracy in the performance of the proposed method in tumor segmentation by its low computational complexity shows the efficiency of the proposed system. Further, the main advantage is its sovereignty from atlas-based registration, earlier anatomical knowledge which restricts the general application of a lot of state-of-the-art methods. All experiments show that this system gives exceptionally better results in comparison with the lately proposed methods. Further, despite several other methods in which there is the need for initial assumptions, the proposed method here does not require any such input. This makes the method much more appealing and common than other methods. The authors have achieved significant accuracy in terms of sensitivity, specificity and DSC at time of extracting tumorous image and at the time of localization. As per the future work, the authors are working on the time complexity of the method via current tools such as CUDA and GPU.

\section{ACKNOWLEDGMENT}

All authors declared no conflict of interest concerning the work in this article. Bhubaneswar is acknowledged for technical and financial support. Brain tumor image data used in this article were obtained from the MICCAI 2017 Challenges on Multimodal Brain Tumor Segmentation. The challenge database contains fully anonymized images from the Cancer Imaging Atlas Archive and the BRATS challenge. 


\section{REFERENCES}

Abd-Ellah, M., Awad, A., Khalaf, A., \& Hamed, H. (2018). Two-phase multi-model automatic brain tumor diagnosis system from magnetic resonance images using convolutional neural networks. EURASIP Journal On Image And Video Processing, 2018(1). 10.1186/s13640-018-0332-4

Abdel-Maksoud, E., Elmogy, M., \& Al-Awadi, R. (2015). Brain tumor segmentation based on a hybrid clustering technique. Egyptian Informatics Journal, 16(1), 71-81. doi:10.1016/j.eij.2015.01.003

Ahmed, S., Iftekharuddin, K., \& Vossough, A. (2011). Efficacy of Texture, Shape, and Intensity Feature Fusion for Posterior-Fossa Tumor Segmentation in MRI. IEEE Transactions on Information Technology in Biomedicine, 15(2), 206-213. doi:10.1109/TITB.2011.2104376 PMID:21216716

Ain, Q., Jaffar, M., \& Choi, T. (2014). Fuzzy anisotropic diffusion-based segmentation and texture based ensemble classification of brain tumor. Applied Soft Computing, 21, 330-340. doi:10.1016/j.asoc.2014.03.019

Al-Janobi, A. (2001). Performance evaluation of cross-diagonal texture matrix method of texture analysis. Pattern Recognition, 34(1), 171-180. doi:10.1016/S0031-3203(99)00206-X

Al-Shaikhli, S., Yang, M., \& Rosenhahn, B. (2014). Multi-region labeling and segmentation using a graph topology prior and atlas information in brain images. Computerized Medical Imaging and Graphics, 38(8), 725-734. doi:10.1016/j.compmedimag.2014.06.008 PMID:24998760

Alqazzaz, S., Sun, X., Yang, X., \& Nokes, L. (2019). Automated brain tumor segmentation on multi-modal MR image using SegNet. Computational Visual Media, 5(2), 209-219. doi:10.1007/s41095-019-0139-y

Angulakshmi, M., \& Lakshmi Priya, G. (2017). Automated brain tumor segmentation techniques- A review. International Journal of Imaging Systems and Technology, 27(1), 66-77. doi:10.1002/ima.22211

Aslam, A., Khan, E., \& Beg, M. (2015). Improved Edge Detection Algorithm for Brain Tumor Segmentation. Procedia Computer Science, 58, 430-437. doi:10.1016/j.procs.2015.08.057

Chuang, K. S., Tzeng, H. L., Chen, S., Wu, J., \& Chen, T. J. (2006). Fuzzy c-means clustering with spatial information for image segmentation. Computerized Medical Imaging and Graphics, Elsevier, 30(1), 9-15. doi:10.1016/j.compmedimag.2005.10.001 PMID:16361080

Clark, M., Hall, L., Goldgof, D., Velthuizen, R., Murtagh, F. R., \& Silbiger, M. S. (1998). Automatic tumor segmentation using knowledge-based techniques. IEEE Transactions on Medical Imaging, 17(2), $187-201$. doi:10.1109/42.700731 PMID:9688151

Dahab, Ghoniemy, Gamal, \& Selim. (2012). Automated brain tumor detection and identification using image processing and probabilistic neural network techniques. Int J Image Process Visual Commun.

El-Dahshan, E., Mohsen, H., Revett, K., \& Salem, A. (2014). Computer-aided diagnosis of human brain tumor through MRI: A survey and a new algorithm. Expert Systems with Applications, 41(11), 5526-5545. doi:10.1016/j. eswa.2014.01.021

Elnakib, A., Gimel'Farb, G., Suri, J.S., \& El-Baz, A. (2011). Medical Image Segmentation: A Brief Survey. Multi Modality State-of-the-Art Medical Image Segmentation and Registration Methodologies, 1-39. 10.1007/9781-4419-8204-9_1

Havaei, M., Dutil, F., \& Pal, C. (2016). A Convolutional Neural Network Approach to Brain Tumor Segmentation. Brainlesion: Glioma, Multiple Sclerosis, Stroke and Traumatic Brain Injuries Lecture Notes in Computer Science, 195-208. doi:10.1007/978-3-319-30858-6_17

Havaei, M., Davy, A., Warde-Farley, D., Biard, A., Courville, A., Bengio, Y., Pal, C., Jodoin, P.-M., \& Larochelle, H. (2017). Brain tumor segmentation with Deep Neural Networks. Medical Image Analysis, 35, 18-31. doi:10.1016/j.media.2016.05.004 PMID:27310171

Stanford Computer Vision Lab. (n.d.). Retrieved from http://vision.stanford.edu/

Islam, A., Reza, S., \& Iftekharuddin, K. (2013). Multifractal Texture Estimation for Detection and Segmentation of Brain Tumors. IEEE Transactions on Biomedical Engineering, 60(11), 3204-3215. doi:10.1109/ TBME.2013.2271383 PMID:23807424 
Işın, A., Direkoğlu, C., \& Şah, M. (2016). Review of MRI-based Brain Tumor Image Segmentation Using Deep Learning Methods. Procedia Computer Science, 102, 317-324. doi:10.1016/j.procs.2016.09.407

Jaffar, M. A., Naveed, N., \& Ahmed, B. (2009). Fuzzy c-means clustering with spatial information for color image segmentation. 2009 Third International Conference on Electrical Engineering. doi:10.1109/ICEE.2009.5173186

Joshi, D. M., Rana, N. K., \& Misra, V. M. (2010). Classification of Brain Cancer using Artificial Neural Network. 2010 2nd International Conference on Electronic Computer Technology. doi:10.1109/ICECTECH.2010.5479975

Kumari, N., \& Saxena, S. (2018). Review of Brain Tumor Segmentation and Classification. 2018 International Conference on Current Trends towards Converging Technologies (ICCTCT). doi:10.1109/ICCTCT.2018.8551004

Lahmiri, S. (2017). Glioma detection based on multi-fractal features of segmented brain MRI by particle swarm optimization techniques. Biomedical Signal Processing and Control, 31, 148-155. doi:10.1016/j.bspc.2016.07.008

Liew, A.-C., \& Yan, H. (2003). An adaptive spatial fuzzy clustering algorithm for 3-D MR image segmentation. IEEE Transactions on Medical Imaging, 22(9), 1063-1075. doi:10.1109/TMI.2003.816956 PMID:12956262

Madhukumar, S., \& Santhiyakumari, N. (2015). Evaluation of k-Means and fuzzy C-means segmentation on MR images of brain. The Egyptian Journal of Radiology and Nuclear Medicine, 46(2), 475-479. doi:10.1016/j. ejrnm.2015.02.008

Menze, B., Jakab, A., Bauer, S., Kalpathy-Cramer, J., Farahani, K., Kirby, J., Burren, Y., Porz, N., Slotboom, J., Wiest, R., Lanczi, L., Gerstner, E., Weber, M.-A., Arbel, T., Avants, B. B., Ayache, N., Buendia, P., Collins, D. L., Cordier, N., \& Van Leemput, K. et al. (2015). The Multimodal Brain Tumor Image Segmentation Benchmark (BRATS). IEEE Transactions on Medical Imaging, 34(10), 1993-2024. doi:10.1109/TMI.2014.2377694 PMID:25494501

Nabizadeh, N., \& Kubat, M. (2015). Brain tumors detection and segmentation in MR images: Gabor wavelet vs. statistical features. Computers \& Electrical Engineering, 45, 286-301. doi:10.1016/j.compeleceng.2015.02.007

Naceur, M. B., Saouli, R., Akil, M., \& Kachouri, R. (2018). Fully Automatic Brain Tumor Segmentation using End-To-End Incremental Deep Neural Networks in MRI images. Computer Methods and Programs in Biomedicine, 166, 39-49. doi:10.1016/j.cmpb.2018.09.007 PMID:30415717

Pereira, S., Pinto, A., Alves, V., \& Silva, C. (2016). Brain Tumor Segmentation Using Convolutional Neural Networks in MRI Images. IEEE Transactions on Medical Imaging, 35(5), 1240-1251. doi:10.1109/ TMI.2016.2538465 PMID:26960222

Pingale, D., \& Todmal, S. (2018). Brain Tumor Segmentation Using K-Means and Fuzzy C-Means Clustering and Its Area Calculation and Stage Using SVM Algorithm. Journal of Advances and Scholarly Researches in Allied Education, 15(3), 27-32. doi:10.29070/15/56750

Sachdeva, J., Kumar, V., Gupta, I., Khandelwal, N., \& Ahuja, C. K. (2016). A package-SFERCB-“Segmentation, feature extraction, reduction and classification analysis by both SVM and ANN for brain tumors. Applied Soft Computing, 47, 151-167. doi:10.1016/j.asoc.2016.05.020

Saxena, S., Garg, A., \& Mohapatra, P. (2019). Advanced Approaches for Medical Image Segmentation. Application of Biomedical Engineering in Neuroscience, 153-172. 10.1007/978-981-13-7142-4_8

Saxena, S., Mohapatra, P., \& Pattnaik, S. (2019). Brain Tumor and Its Segmentation From Brain MRI Sequences. Early Detection of Neurological Disorders Using Machine Learning Systems Advances in Medical Technologies and Clinical Practice, 39-60. doi:10.4018/978-1-5225-8567-1.ch004

Saxena, S., Paul, S., Garg, A., Saikia, A., \& Datta, A. (2020). Deep Learning in Computational Neuroscience. Advances in Computer and Electrical Engineering, 43-63. doi:10.4018/978-1-7998-0182-5.ch002

Soltaninejad, M., Yang, G., Lambrou, T., Allinson, N., Jones, T. L., Barrick, T. R., Howe, F. A., \& Ye, X. (2016). Automated brain tumor detection and segmentation using superpixel-based extremely randomized trees in FLAIR MRI. International Journal of Computer Assisted Radiology and Surgery, 12(2), 183-203. doi:10.1007/ s11548-016-1483-3 PMID:27651330

Sonavane, R., \& Sonar, P. (2016). Classification and segmentation of brain Tumor using Adaboost classifier. 2016 International Conference On Global Trends In Signal Processing, Information Computing And Communication (ICGTSPICC). doi:10.1109/ICGTSPICC.2016.7955334 
Subbanna, N., Precup, D., \& Arbel, T. (2014). Iterative Multilevel MRF Leveraging Context and Voxel Information for Brain Tumor Segmentation in MRI. 2014 IEEE Conference on Computer Vision and Pattern Recognition. doi:10.1109/CVPR.2014.58

Sudharani, K., Sarma, T., \& Prasad, K. (2016). Advanced Morphological Technique for Automatic Brain Tumor Detection and Evaluation of Statistical Parameters. Procedia Technology, 24, 1374-1387. doi:10.1016/j. protcy.2016.05.153

Telrandhe, S. R., Pimpalkar, A., \& Kendhe, A. (2016) Detection of brain tumor from MRI images by using segmentation \& SVM. 2016 World Conference on Futuristic Trends in Research and Innovation for Social Welfare (Startup Conclave). doi:10.1109/STARTUP.2016.7583949

Viswa Priya, V., \& Shobarani, . (2016). An Efficient Segmentation Approach for Brain Tumor Detection in MRI. Indian Journal of Science and Technology, 9(19). Advance online publication. doi:10.17485/ijst/2016/v9i19/90440

Quick Brain Tumor Facts. (2018). Retrieved 8 December 2018 from https://braintumor.org/brain-tumorinformation/brain-tumor-facts/

Basics, M. R. I. (2018). Retrieved 8December 2018, from http://casemed.case.edu/clerkships/neurology/Web\%20 Neurorad/MRI\%20Basics.htm

Sanjay Saxena's research interests are in the field of Medical Image Processing, High Performance Computing, Deep Learning. Dr. Saxena teaches Image Processing, Java Programming, Computer Vision and Parallel Computing. Dr. Saxena has published almost 27 research articles in various peer reviewed international \& national journals and conferences. Dr. Saxena is the member of various international societies such as IEEE, SCIEI, ACM, IAENG, ISOC. Dr. Saxena has visited several national institutes for the presentation and invited talk on significant research areas of parallel medical image processing. He has also visited numerous international technically recognized institutes like Imperial College London, United Kingdom, Vienna University of Technology, Austria, Stonybrook University, New York, USA.

Nitu Kumari has completed her B. Tech in Information Technology from Dr. A.P.J. Abdul Kalam Women's Institute of Technology, Darbhanga, Bihar and M. Tech from Computer Science \& Engineering from IIIT Bhubaneswar.

Swati Pattnaik is an Information Technology student from International Institute of Information Technology, Bhubaneswar. Her research interest lies in the field of Data Science, Machine Learning, Image Processing and Natural Language Processing. 